\title{
Relação entre força dinâmica máxima de membros inferiores e o equilíbrio corporal em praticantes de musculação
}

\author{
Relationship between maximum dynamic force of inferior members \\ and body balance in strength training apprentices
}

Ariane Martins

Érico Felden Pereira

Clarissa Stefani Teixeira

Sara Teresinha Corazza

1 Universidade Federal de Santa Maria, Santa Maria, RS. Brasil.

2 Universidade Federal do Paraná, Curitiba, PR. Brasil.

3 Universidade Federal de Santa Catarina, Florianópolis, SC. Brasil.

Recebido em 21/07/09 Revisado em 23/08/09. Aprovado em 22/01/10
Resumo - A relação entre força e equilíbrio apresenta resultados controversos e possui implicações diretas na prática de prescrição de exercícios. O objetivo do estudo foi investigar e relação entre a força dinâmica máxima (FDM) de membros inferiores e os equilíbrios estáticos e dinâmicos. Participaram do estudo 60 indivíduos, de 18 a 24 anos, praticantes de musculação. Para avaliação da FDM foi utilizado o teste de 1 Repetição Máxima (1RM) para dois aparelhos: leg press e extensão de joelhos. Foram aplicados testes motores para avaliar os equilíbrios estáticos e dinâmicos. Massa corporal, estatura e tempo de prática foram controlados. As associações entre as variáveis foram analisadas por meio de testes de correlação e análises de regressão linear. Os resultados indicaram correlações significativas entre força e equilíbrio estático apenas para o gênero feminino $(\mathrm{p}=0,038)$. Para o gênero masculino, observou-se correlação significa entre a massa corporal e o equilíbrio estático $(\mathrm{p}=0,045)$. A capacidade explicativa da FDM e do tempo de prática na musculação para o desempenho nos testes de equilíbrio foram baixas: $13 \%$ para o equilíbrio estático no gênero masculino e de $18 \%$ e 17\%, para o estático e o dinâmico, no gênero feminino. Em conclusão a FDM de membros inferiores apresentou baixa capacidade preditiva para desempenho nos equilíbrios estático e dinâmico, principalmente, para os homens.

Palavras-chave: Equilíbrio musculoesquelético; Força muscular; Desempenho psicomotor

Abstract - The relationship between force and balance show controversy results and has direct implications in exercise prescription practice. The objective was to investigate the relationship between maximum dynamic force (MDF) of inferior limbs and the static and dynamic balances. Participated in the study 60 individuals, with 18 to 24 years old, strength training apprentices. The MDF was available by mean the One Maximum Repetition (IMR) in "leg press" and "knee extension" and motor testes to available of static and dynamic balances. The correlation tests and multiple linear regression were applied. The force and balance variables showed correlation in females $(p=0.038)$. The corporal mass and static balance showed correlation for the males $(p=0.045)$. The explication capacity at MDF and practices time were small: $13 \%$ for static balance in males, $18 \%$ and $17 \%$, respectively, for static and dynamic balance in females. In conclusion: the MDF of inferior limbs showed low predictive capacity for performance in static and dynamic balances, especially for males.

Key words: Musculoskeletal equilibrium; Muscle strength; Psychomotor performance. 


\section{INTRODUÇÃO}

Desde os primeiros meses de vida, os indivíduos são instigados, naturalmente, a desempenhar tarefas de movimento, como engatinhar, sentar de forma autônoma e, posteriormente, ficar em pé, caminhar e a correr. Para que essas ações sejam realizadas, o desenvolvimento de um conjunto de capacidades motoras é necessário ${ }^{1}$. Embora as capacidades motoras se apresentem de forma independente no ser humano, alterações no sistema neuromuscular e na produção de força podem desencadear perturbações no equilíbrio corporal ${ }^{2-4}$.

A capacidade motora equilíbrio é importante para o desempenho de inúmeras tarefas de movimento e pode sofrer influências de fatores, tanto de origem central, como periférica ${ }^{5}$, uma vez que o sistema nervoso regula a estabilidade corporal durante a locomoção e o equilíbrio, usando as informações provenientes das estruturas vestibulares ${ }^{6}$, do sistema visual ${ }^{7}$ e do sistema proprioceptivo ${ }^{4,8}$.

Barela ${ }^{9}$ discute que oscilações constantes ocorrem mesmo quando uma pessoa procura manter-se em pé o mais estável possível. Essas oscilações são decorrentes da dificuldade em manter os muitos segmentos corporais alinhados entre si sobre uma base de suporte restrita, utilizando-se do sistema muscular que produz forças que variam ao longo do tempo. Além disso, a manutenção da estabilidade corporal está relacionada ao balanceamento entre forças internas e externas, que agem no corpo durante a realização de ações motoras.

Observa-se na literatura carência de estudos que busquem identificar a relação entre força dinâmica máxima (FDM) de membros inferiores, que é uma das capacidades motoras mais treinadas nas academias de ginástica, e o equilíbrio corporal em indivíduos adultos saudáveis, praticantes ou não de exercícios. Neste contexto, buscou-se analisar a relação entre a FDM de membros inferiores e o desempenho em testes de equilíbrio estático e dinâmico, em indivíduos adultos, com a intenção de contribuir para a compreensão da relação entre as diferentes capacidades motoras e vislumbrar possíveis alternativas de intervenção para a melhoria do equilíbrio.

\section{PROCEDIMENTOS METODOLÓGICOS}

\section{Amostra}

A amostra foi formada por 30 indivíduos do gênero masculino e 30 do gênero feminino, com idades entre 18 a 24 anos, praticantes de musculação em duas academias na cidade de Santa Maria, RS, Brasil. Estabeleceu-se como critério de inclusão, além da idade, ter realizado, no mínimo, oito sessões do treino de musculação, para que pudessem realizar o teste de 1RM, conforme protocolo utilizado por Moura e Zinn ${ }^{10}$, de forma que este não viesse causar lesões e, no máximo, 60 sessões ou três meses de prática, para minimizar as diferenças em função do tempo de prática.

Todos os voluntários foram informados das características do trabalho e assinaram o Termo de Consentimento Livre e Esclarecido. Este estudo foi aprovado pela Comissão de Ética em Pesquisa (CEP) da Universidade Federal de Santa Maria (UFSM), conforme determina a resolução 196/96 do Conselho Nacional de Saúde (CNS) e está registrado sob o número 0096.0.243.000-06.

\section{Instrumentos e procedimentos}

Inicialmente, foi aplicada uma ficha de anamnese, por meio da qual foram coletados dados pessoais, informações sobre tempo de prática, presença ou não de fraturas ou lesões nos membros inferiores e ainda, questões relacionadas a doenças associadas ao sistema visual e ao sistema proprioceptivo, sendo excluídos da amostra aqueles sujeitos que relataram quaisquer dessas situações. Além disso, considerando que, conforme destacam Bankoff et al. ${ }^{5}$, os sujeitos precisam estar funcionalmente entrosados para a boa manutenção do equilíbrio, foi permitido aos mesmos que experimentassem as posições de equilíbrio que seriam avaliadas antes da testagem.

Além das informações na anamnese, foram coletadas as seguintes variáveis: força dinâmica máxima para "pressão de perna" (PP) e para extensão de joelho (EJ); equilíbrio estático (EE) e dinâmico (ED), tempo de prática (TP), massa corporal (MC) e estatura. Para a avaliação da força dinâmica máxima de membros inferiores, foi realizado o teste de 1RM que apresenta os seguintes índices de fidedignidade, validade e objetividade, respectivamente: 0,$977 ; 0,839$ e 0,960 para a PP e 0,$984 ; 0,755$ e 0,985 para a $\mathrm{EJ}^{10}$.

O teste foi realizado nos aparelhos leg press e cadeira extensora de joelhos que eram do mesmo modelo e marca nas duas academias investigadas. O tempo de intervalo de um aparelho para o outro variou de três a cinco minutos de descanso, a fim de evitar a possível fadiga muscular, assim como o número de tentativas que foram, no máximo, cinco, para não ultrapassar cinco alterações de cargas, conforme o protocolo ${ }^{10}$. Todos os testes foram realizados no mesmo dia, primeiramente, era 
realizado o teste de equilíbrio estático e após, o de equilíbrio dinâmico e finalmente, o teste de 1RM (leg press e cadeira extensora).

Para avaliar o equilíbrio dinâmico foi utilizado o "The Nelson Balance Test"11. O teste tem duas fases, na $1^{\mathrm{a}}$ fase, quando o testando estiver pronto, ele deve dar um passo em cima do primeiro bloco, com o membro inferior esquerdo, utilizando a ponta do pé. Há quatro blocos diferentes em que o testando deve permanecer em equilíbrio em um só membro por 5 segundos. Quando andar pela trave de equilíbrio, ele deve caminhar até a primeira metade da trave; quando atingir esta metade, deverá executar um giro de 180 graus e caminhar lateralmente para a direita até o final. $\mathrm{Na} 2^{\mathrm{a}}$ fase, tão logo o testando saia do último bloco, ele deverá executar um giro e retornar ao bloco, repetindo todo procedimento da $1^{\text {a }}$ fase. $\mathrm{O}$ resultado será o tempo cumulativo dado em décimos de segundos para as duas fases do teste.

Para avaliar o equilíbrio estático, foi utilizado o "Stork Stand Test"11, no qual o indivíduo fica parado sobre o membro inferior dominante e posiciona o pé contralateral na parte medial do joelho de apoio. As mãos ficam nos quadris e ao ser dado um sinal o indivíduo tira o calcanhar do pé de apoio do chão; o equilíbrio deve ser mantido o maior tempo possível sem mover a parte que apóia o pé de sua posição inicial ou deixar o calcanhar tocar o chão. São dadas três tentativas; o resultado é o tempo do equilíbrio mais longo.

Foram realizadas análises descritivas considerando homens e mulheres separadamente, cujas diferenças foram analisadas por meio do teste $t$ de Student. O teste de correlação de Spearman foi aplicado, buscando identificar a correlação entre as variáveis PP, EJ em relação ao desempenho nos testes de equilíbrio e, o teste de Correlação de Pearson entre as variáveis MC e estatura em relação ao desempenho no equilíbrio. Além disso, foi realizada análise de regressão linear múltipla entre as variáveis $\mathrm{MC}$, estatura, PP, EJ em relação aos desempenhos de equilíbrio. A normalidade dos dados e do resíduo do modelo de regressão ajustado foi confirmada por meio do teste Shapiro-Wilk. Para todas as análises, foi adotado um nível de significância de 5\%.

\section{RESULTADOS}

As análises mostraram diferenças significativas na FDM entre homens e mulheres $(p<0,001)$ tanto para a variável PP como para a EJ. Já na análise do equilíbrio estático e dinâmico não foram observa- das diferenças estatisticamente significativas entres os gêneros (Tabela 1). Os resultados das correlações mostraram associações baixas e moderadas para a maior parte das variáveis (Tabelas 2 e 3). Para os homens, a massa corporal apresentou correlação significativa com o EE $(p=0,045)$ e para as mulheres o EE esteve correlacionado com a PP $(p=0,016)$ e com a PJ $(p=0,038)$. Considerando o ED, observouse correlação significativa com a PP $(\mathrm{p}=0,024)$.

Tabela 1. Dados descritivos do grupo pesquisado e teste de diferença entre gêneros.

\begin{tabular}{lccc}
\hline \multirow{2}{*}{ Variáveis } & Masculino & Feminino & \multirow{2}{*}{ p-valor* } \\
\cline { 2 - 3 } & Média (DP) & Média (DP) & \\
\hline Idade (anos) & $20,16(1,78)$ & $21,66(2,47)$ & 0,009 \\
MC (kg) & $73,90(8,83)$ & $58,23(5,51)$ & $<0,001$ \\
Estatura (m) & $1,77(0,005)$ & $1,65(0,005)$ & $<0,001$ \\
PP (kg) & $121,87(26,39)$ & $82,17(27,74)$ & $<0,001$ \\
EJ (kg) & $62,07(15,10)$ & $42,80(15,89)$ & $<0,001$ \\
EE (s) & $18,00(12,78)$ & $20,66(16,83)$ & 0,491 \\
ED (s) & $72,22(13,11)$ & $76,87(13,25)$ & 0,177 \\
TP (meses) & $1,93(0,73)$ & $1,90(0,92)$ & 0,878 \\
\hline
\end{tabular}

* probabilidade do teste $t$ de Student

MC: massa corporal; PP: pressão de pernas; EJ: extensão de joelhos; EE: equilíbrio estático; ED: equilíbrio dinâmico; TP: tempo de prática.

Tabela 2. Correlações entre massa corporal, estatura, força dinâmica máxima e tempo de prática com os desempenhos de equilíbrio para o grupo masculino.

\begin{tabular}{lcccc}
\hline \multirow{2}{*}{ Variável } & \multicolumn{2}{c}{ EE } & \multicolumn{2}{c}{ ED } \\
\cline { 2 - 5 } & $\mathrm{r}$ & $\mathrm{p}$ & $\mathrm{r}$ & $\mathrm{P}$ \\
\hline MC & 0,369 & 0,045 & $-0,138$ & 0,468 \\
Estatura & 0,251 & 0,182 & $-0,187$ & 0,323 \\
PP & 0,215 & 0,255 & $-0,102$ & 0,590 \\
EJ & 0,309 & 0,960 & $-0,278$ & 0,136 \\
TP & 0,173 & 0,362 & $-0,027$ & 0,887 \\
\hline
\end{tabular}

* correlação de Pearson para as variáveis contínuas e correlação de Spearman para variáveis categóricas. PP: pressão de pernas; EJ: extensão de joelhos; EE: equilíbrio estático; ED: equilíbrio dinâmico; TP: tempo de prática

Tabela 3. Correlações entre massa corporal, estatura, força dinâmica máxima e tempo de prática com os desempenhos de equilíbrio para o grupo feminino.

\begin{tabular}{lcccc}
\hline \multirow{2}{*}{ Variável } & \multicolumn{2}{c}{ EE } & \multicolumn{2}{c}{ ED } \\
\cline { 2 - 5 } & $r$ & $p$ & $r$ & $P$ \\
\hline MC & 0,206 & 0,275 & 0,181 & 0,311 \\
Estatura & 0,148 & 0,405 & 0,041 & 0,828 \\
PP & 0,436 & 0,016 & $-0,412$ & 0,024 \\
EJ & 0,381 & 0,038 & $-0,280$ & 0,052 \\
TP & 0,238 & 0,206 & $-0,170$ & 0,370 \\
\hline
\end{tabular}

* correlação de Pearson para as variáveis contínuas e correlação de Spearman para variáveis categóricas. PP: pressão de pernas; El: extensão de joelhos; EE: equilíbrio estático; ED: equilíbrio dinâmico; TP: tempo de prática. 
Nas análises de regressão linear, foram testados modelos completos, ou seja, incluindo todas as variáveis investigadas. No entanto, foram apresentados nas Tabelas 4 e 5 apenas os modelos com variáveis que apresentaram associações significativas com o equilíbrio nos modelos ajustados.

Considerando o equilíbrio estático no grupo masculino pelo modelo de análise de regressão linear calculado pelo modo Stepwise, todas as variáveis, com exceção da massa corporal, foram retiradas do modelo. A massa corporal, neste modelo, foi a única que apresentou relação significativa (Tabela 4). No entanto, sua capacidade de explicar o desempenho de equilíbrio foi baixa, aproximadamente 13\%. Com relação ao equilíbrio dinâmico, no grupo masculino, nenhuma variável investigada apresentou capacidade significativa de explicar os resultados do equilíbrio não sendo possível, neste caso, apresentar valores de coeficiente de determinação $\left(\mathrm{R}^{2}\right)$.

Considerando o equilíbrio estático no grupo feminino através do modo Stepwise, todas as variáveis, com exceção da PP, foram retiradas do modelo de regressão. A PP nesse modelo foi a única que apresentou relação significativa em ambos os equilíbrios avaliados. No entanto, sua capacidade de explicar o desempenho de equilíbrio foi baixa, explicando apenas $18 \%$ dos resultados. Em relação ao equilíbrio dinâmico no grupo feminino, os dados tiveram o mesmo comportamento, sendo que, neste caso, a PP explicou $17 \%$ do desempenho do equilíbrio, conforme dados apresentados na Tabela 4.

Tabela 4. Resultados da análise de regressão, considerando as variáveis investigadas.

\begin{tabular}{|c|c|c|c|c|}
\hline & \multicolumn{4}{|c|}{ Masculino } \\
\hline \multirow[b]{2}{*}{ Variáveis } & \multicolumn{4}{|c|}{$\mathrm{EE}$} \\
\hline & \multicolumn{2}{|c|}{ Coeficientes $\beta$} & \multicolumn{2}{|c|}{ p-valor* } \\
\hline \multirow[t]{3}{*}{ MC } & \multicolumn{2}{|c|}{0,534} & \multicolumn{2}{|c|}{0,045} \\
\hline & \multicolumn{4}{|c|}{$\mathrm{R}^{2}=0,136$} \\
\hline & \multicolumn{4}{|c|}{ Feminino } \\
\hline \multirow{2}{*}{ Variáveis } & \multicolumn{2}{|l|}{$\mathrm{EE}$} & \multicolumn{2}{|l|}{$\mathrm{ED}$} \\
\hline & Coeficiente $\beta$ & p-valor* & Coeficiente $\beta$ & p-valor* \\
\hline \multirow[t]{2}{*}{ PP } & 0,260 & 0,018 & $-0,198$ & 0,022 \\
\hline & \multicolumn{2}{|c|}{$R^{2}=0,184$} & \multicolumn{2}{|c|}{$R^{2}=0,172$} \\
\hline
\end{tabular}

* probabilidade do teste t de Student.

EE: equilíbrio estático; $M C$ : massa corporal; ED: equilíbrio dinâmico; PP: pressão de pernas.

\section{DISCUSSÃO}

As variáveis analisadas neste estudo incluíram as capacidades motoras, FDM de membros inferiores e o equilíbrio corporal (dinâmico e estático) em adultos. Os estudos com as variáveis citadas têm sido conduzidos, especialmente, com análises de riscos de queda em idosos ${ }^{12-14}$, o que demonstra uma lacuna de conhecimento com outras faixas etárias, especialmente, adultos. Estudos conduzidos com idosos $^{15}$ verificaram, por exemplo, que o risco de incapacidade de andar foi 10 vezes maior em idosos com alterações na força muscular e no equilíbrio do que quando comparados àqueles com alteração em somente uma dessas capacidades.

Embora não se tenham encontrados estudos com relações entre a FDM e o equilíbrio em adultos, análises do efeito da prática de exercícios físicos, especificamente relacionado ao treinamento de força, têm sido evidenciadas pela literatura ${ }^{16,17}$. $\mathrm{O}$ estudo de Aveiro et al. ${ }^{16}$ descreveu os efeitos de um programa de treinamento de 12 semanas, em mulheres jovens, formado de exercícios de alongamento, caminhada, fortalecimento dos músculos do quadril e treinamento de equilíbrio, sendo a avaliação do equilíbrio realizada por meio de testes de equilíbrio estático e dinâmico. Os resultados mostraram que, similarmente ao aumento de força muscular, houve também melhoria significativa no desempenho de equilíbrio após o treinamento.

Pant et al ${ }^{17}$ verificaram a correlação entre a força muscular (na dorsiflexão, flexão plantar, inversão e eversão do tornozelo) e o equilíbrio corporal de 20 indivíduos do gênero masculino, entre 19 e 21 anos de idade. O equilíbrio apresentou correlações significativas com dorsiflexão $(\mathrm{r}=0,638)$ e com flexão plantar $(\mathrm{r}=0,443)$ e não mostrou correlações significativas com eversão $(\mathrm{r}=0,203)$ e inversão $(\mathrm{r}=0,159)$. De maneira geral, os autores concluíram que os indivíduos que possuem boa força muscular no tornozelo possuem também um melhor equilíbrio corporal, tendência que deve ser observada com cautela no presente estudo.

Considerando os resultados encontrados nos sujeitos avaliados, verificou-se diferença significativa em favor das mulheres nos testes de equilíbrio, o que vai ao encontro, por exemplo, do estudo de Ilkiv ${ }^{18}$ que mostrou uma tendência das mulheres apresentarem maiores estabilidades que os homens. $\mathrm{O}$ autor avaliou 22 mulheres e 18 homens, com idade acima de 60 anos, participantes a 12 meses de um programa de atividades físicas (vôlei, hidroginástica, bocha e recreação) e analisou o equilíbrio estático com controle visual e apoio unipodal, identificando índices superiores de equilíbrio para as mulheres.

Com relação aos homens, houve correlação significativa entre a massa corporal e o equilíbrio estático. Hue et al. ${ }^{19}$ discutem que a massa corporal é uma das variáveis que mais explica as variações no 
equilíbrio. Corbeil et al. ${ }^{20}$ encontraram um aumento no torque muscular do tornozelo para a manutenção do equilíbrio de indivíduos com maior massa corporal. Os autores relacionaram o fato de o aumento de massa corporal aumentar a quantidade de movimento do centro de massa que, por sua vez, obriga uma maior produção de força dos indivíduos com maior massa corporal para o controle postural.

No caso da musculação, pode ocorrer um aumento da massa corporal magra o que, consequentemente, requer músculos mais fortalecidos. Logo, indivíduos com estas características, conforme discutem Hamaoui et al. ${ }^{21}$, apresentam um melhor controle neuromuscular e consequentemente, menor dificuldade para manter a estabilidade do corpo. Nas mulheres, a associação entre o desempenho de força no exercício pressão de perna e os equilíbrios estático e dinâmico e a não associação com a massa corporal podem remeter à necessidade de análises que fragmentam o peso corporal em massa gorda e magra.

As baixas capacidades explicativas da força para os equilíbrios podem ter sofrido influências do treinamento. Bellew et al. ${ }^{22}$ discutem que exercícios que não são realizados em pé como, por exemplo, o leg press e o extensor de joelhos, podem não refletir em melhorias significativas da estabilidade corporal. Assim, estudos experimentais que analisem força e equilíbrio em diferentes treinamentos de musculação podem colaborar para elucidação do tema.

Importante salientar, também, que os proprioceptores, fuso muscular, no músculo, e os órgãos tendinosos de golgi, no tendão, são considerados como sendo os órgãos sensoriais que monitoram o estado do sistema músculoesquelético ${ }^{23}$. Logo, ao se treinar a musculatura, por meio de treinamentos de força como na musculação, estes órgãos estarão sendo ativados e treinados. Apesar disso, uma correlação direta dessa possível melhoria neuromotora proporcionada pelos exercícios resistidos e o equilíbrio deve ser pensada com cautela de acordo com os resultados apresentados.

A principal limitação apontada neste estudo está relacionada ao método de avaliação do equilíbrio. Embora se tenha utilizado testes motores padronizados e validados, em função do ambiente no qual seriam realizadas as avaliações, futuros estudos semelhantes podem ser conduzidos com avaliações mais precisas como plataformas de força e posturografia.

\section{CONCLUSÕES}

As correlações entre FDM e os equilíbrios estático e dinâmico parecem se comportar de forma diferente para os sujeitos dos gêneros masculino e feminino, sendo essa associação mais significativa nas mulheres. A capacidade preditiva da FDM para os desempenhos nos testes de equilíbrio foi baixa, remetendo à necessidade de treinamentos individuais para as duas capacidades motoras em adultos. Análises de diferentes tipos de treinamento e da relação entre equilíbrio e força em outras faixas etárias e em sujeitos sedentários e ativos podem ajudar na clarificação do tema.

\section{REFERÊNCIAS BIBLIOGRÁFICAS}

1. Gallahue DL. A classificação das habilidades de movimento: um caso para modelos multidimensionais. Rev Educ Fís/UEM 2002;13(2):105-11

2. Takala EP, Korhonen L, Viikari-Juntura E. Postural sway and stepping response among working population: reproducibility, long-term stability, and associations with symptoms of the low back. Clin Biomech 1997;12(7-8):429-37.

3. Tresch MC. A balanced view of motor control. Nat Neurosci 2007;10:1127-8.

4. Mademli L, Arampatzis A, Karamanidis K. Dynamic stability control in forward falls: postural corrections after muscle fatigue in young and older adults. Eur J Appl Physiol 2008;103(3):295-306.

5. Bankoff ADP, Bekedorf RG, Schmidt A, Ciol P, Zamai CA. Análise do equilíbrio corporal estático através de um baropodômetro eletrônico. Rev Conexões 2006;4(2):19-30.

6. Bacsi AM, Colebatch JG. Evidence for reflex and perceptual vestibular contributions to postural control. Exp Brain Res 2005;160(1):22-8.

7. Mergner T, Schweigart G, Maurer C, Blümle A. Human postural responses to motion of real and virtual visual environments under different support base conditions. Exp Brain Res 2005;167(4):535-56.

8. Bove M, Nardone A, Schieppati M. Effects of leg muscle tendon vibration on group Ia and group II reflex responses to stance perturbation in humans. J Physiol 2003;550(2):617-30.

9. Barela JA. Estratégias de controle em movimentos complexos: ciclo percepção-ação no controle postural. Rev Paul Educ Fís 2000;supl.3:79-88.

10. Moura JAR, Zinn JL. Proposição e validação de modelos matemáticos regressivos para estimativa da força dinâmica máxima a partir de variáveis preditivas neuromusculares. Rev Bras Cineantropom Desempenho Hum 2002;4(1):25-36.

11. Jonhnson BL, Nelson JK. Pratic measurements for evaluation in physical education. 4 th. Edina: Brugess; 1986.

12. Campbell AJ, Robertson MC, Gardner MM, Norton RN, Tilyard MW, Buchner DM. Randomized controlled trial of a general practice programme of home based exercise to prevent falls in elderly women. BM] 1997;315(7115):1065-69. 
13. Topp R, Estes PK, Dayhoff N, Suhrheinrich J. Postural control and strength and mood among older adults. Appl Nurs Res 1997;10(1):11-8.

14. Chan BK, Marshall LM, Winters KM, Faulkner KA, Schwartz AV, Orwoll ES. Incident fall risk and physical activity and physical performance among older men: the osteoporotic fractures in men study. Am J Epidemiol 2007;165(6):696-703.

15. Rantanem T, Guralnik, JM, Foley D, Masaki, K, Leveille S, Curb JD. Midlife hand strength as a predictor of old age disability. JAMA 1999;281(6):558-60.

16. Aveiro MC, Granito RN, Navega MT, Driusso P, Oishi $\mathrm{J}$. Influence of a physical training program on muscle strength, balance and gait velocity among women with osteoporosis. Rev Bras Fisioter 2006;10(4):441-8.

17. Pant H, Sukumar K, Sharma H, Kumar Pandey A, Goel SN. Correlation between muscles strength in relation to dorsiflexion, planterflextion, eversion \& inversion strength with body balance. J Biomech 2006;39(1):557-63.

18. Ilkiv TF. Avaliação da aptidão física de idosos do centro de convivência da melhor idade do município de Monte Alto. [Dissertação de Mestrado - Programa de Pós-Graduação em Promoção da Saúde]. Franca (SP): Universidade de Franca; 2005.

19. Hue O, Simoneau M, Marcotte J, Berrigan F, Doré J, Marceau P, et al. Body weight is a strong predictor of postural stability. Gait Posture 2007;26(1):32-8.
20. Corbeil P, Simoneau M, Rancourt D, Tremblay A, Teasdale N. Increased risk for falling associated with obesity: mathematical modeling of postural control. IEEE Trans Neural Syst Rehabil Eng 2001;9(2):126-36.

21. Hamaoui A, Le Bozec S, Poupard L, Bouisset S. Does postural chain muscular stiffness reduce postural steadiness in a sitting posture. Gait Posture 2007;25(2):199-204.

22. Bellew JW, Yates JW, Gater DR. The initial effects of low-volume strength training on balance in untrained older men and women. J Strength Conditioning Res 2003;17:121-8.

23. McGinnis PM. Biomecânica do esporte e do exercício. Porto Alegre: Artmed, 2002.

Endereço para correspondência

Érico Felden Pereira

Otacílio Chaves, 253

CEP 97045-360 - Santa Maria RS

E-mail: ericofelden@gmail.com 